\title{
IoT and Fog Computing based Predictive Maintenance Model for Effective Asset Management in Industry 4.0 using Machine Learning
}

\author{
Yyi Kai Teoh, Sukhpal Singh Gill, and Ajith Kumar Parlikad
}

\begin{abstract}
The assets in Industry 4.0 are categorised into physical, virtual and human. The innovation and popularisation of ubiquitous computing enhance the usage of smart devices: RFID tags, QR codes, LoRa tags, etc. for assets identification and tracking. The generated data from Industrial Internet of Things (IIoT) eases information visibility and process automation in Industry 4.0. Virtual assets include the data produced from IIoT. One of the applications of the industrial big data is to predict the failure of manufacturing equipment. Predictive maintenance enables the business owner to decide such as repairing or replacing the component before an actual failure which affects the whole production line. Therefore, Industry 4.0 requires an effective asset management to optimise the tasks distributions and predictive maintenance model. This paper presents the Genetic Algorithm (GA) based resource management integrating with machine learning for predictive maintenance in fog computing. The time, cost and energy performance of GA along with MinMin, MaxMin, FCFS, RoundRobin are simulated in the FogWorkflowsim. The predictive maintenance model is built in two-class logistic regression using real-time datasets. The results demonstrate that the proposed technique outperforms MinMin, MaxMin, FCFS, RoundRobin in execution time, cost and energy usage. The execution time is $0.48 \%$ faster, $5.43 \%$ lower cost and energy usage is $\mathbf{2 8 . 1 0 \%}$ lower in comparison with second-best results. The training and testing accuracy of the prediction model is $95.1 \%$ and $\mathbf{9 4 . 5 \%}$, respectively.
\end{abstract}

Index Terms-Fog computing, Industry 4.0, Internet of Things, predictive maintenance, resource management

\section{INTRODUCTION}

$\mathrm{T}$ HE exponential growth of new generation computing such as cloud edge computing, Internet of Things, big data, cyber-physical system (CPS) etc., contributes substantially in the manufacturing industry to accomplish a more proficient, competing and smart manufacturing. Smart manufacturing represents as a future-condition of manufacturing, where the ongoing transmission and analysis of data from shop floor produces manufacturing knowledge, which has a positive effect over all parts of activities. The Industrial Internet of Things (IIoT) is an extension of the Internet of Things (IoT) to use in industrial sectors. IIoT mainly gathers the massively

Y. K. Teoh and S. S. Gill are with School of Electronic Engineering and Computer Science, Queen Mary University of London, UK (emails: y.teoh@se19.qmul.ac.uk, s.s.gill@qmul.ac.uk) interconnected sensors industrial data at the shop floor to produce information, knowledge and control manufacturing system [1]. The utilisation of IIoT incorporates infrastructure, maintenance, process control and supply chain.

GE intelligent platform reports that a health care product manufacturer creates 5,000 samples each 33 milliseconds, likeness 4 trillion of samples each year [2]. Manufacturing factory with one hundred machines tools and ten cameras generate 72 TB of data per year [3]. Conventional in-house servers with constraining resources, i.e. storage, memory, processing power are not fit for processing the new challenging due to scalability and computational complexity and shall deploy in cloud datacentre. However, simulation research in [4] concludes that cloud data centre potentially experiences higher latency and network usage due to the vast geographical distance between IIoT devices and cloud data centre. Fog computing as an expansion of cloud computing to the edge of system networking consists of cloud and edge resources that reduces the latency and network congestion. Therefore, latencysensitive applications can be executed in fog computing.

A distributed system refers to multiple systems that are interlinked while appearing as a single system to the user to enhance resource sharing [5]. Resource management in a distributed system is a fundamental process that involves resource scheduling and allocating resources to applications [6]. Genetic Algorithm (GA) has been generally applied to improve and optimise the resources allocation, and GA is one of the most dependable and promising metaheuristics [7]. GA is part of the evolutionary algorithm, where it is inspired by the evolutionary theory and nature process selection.

The assets in the manufacturing sector include physical asset, human asset, and virtual asset. The reduction of tag cost and infrastructure cost greatly enhance the usage of RFID, 1D barcodes, QR codes, BLE tag, and LoRa tag in assets management. Open Platform Communications United Architecture (OPC UA) is the primary data exchange standard for industrial communication recommended by Reference Architecture Model for Industry 4.0 (RAMI 4.0). OPC UA allows the primary physical assets (manufacturing equipment)

A. K. Parlikad is with Institute for Manufacturing, University of Cambridge, UK (email: aknp2@cam.ac.uk) 
to communicate with each other or to exchange information with the gateway.

\section{A. Motivation and our contributions}

Industry 4.0 enhances the productivity of manufacturing technologies through collection and analysis of real-time data. The ease of communication in IIoT enables real-time tracking and identification of assets within businesses originating from IIoT sources and information services. Industrial big data generated from IIoT sensors promotes information visibility. One of the critical applications in manufacturing is to predict the condition of manufacturing equipment. Therefore, there is a requirement of an efficient and effective resource management technique to handle the generated data. To this end, Genetic Algorithm (GA) seems a promising approach as GA is favourable in band selection, smaller size classification, training and testing accuracies compared to existing works.

The main contributions of this research paper are:

1. Proposed resource scheduling technique: Genetic Algorithm (GA) for assets management in Industry 4.0.

2. Simulated GA with various scheduling techniques, namely MinMin, MaxMin, FCFS and RoundRobin. The performance metrics are time, cost, and energy.

3. Optimised the Decision Support System (DSS) in the production line by implementing predictive maintenance.

4. Presented a detailed case study of manufacturing equipment predictive maintenance using GA and two-class logistic regression.

5. Proposed promising future directions for this research paper.

\section{B. Article organisation}

The rest of the paper is organised as follows. Section II presents the literature review of existing techniques. Section III describes the proposed resource management technique. The experimental setup and case study are presented in Section IV. Section V presents the results of the evaluation. Section VI concludes the paper and further work.

\section{RELATED WORKS}

Institute of Asset Management (IAM) built up a theoretical model for physical, virtual and human asset management. IAM assembled the six key subjects for assets management, to be specific, (1) strategy and planning, (2) asset management decision making, (3) lifecycle delivery, (4) asset information, (5) organisation and people, and (6) risk \& reviews [8].

Authors proposed a novel classification for multi-unit systems asset management into the fleet and portfolio according to a variety of assets and intervention options [9]. Fleet referred to as a system of homogeneous assets while portfolio was a system of the heterogeneous asset. The dependencies in multiunit systems are categorised into performance, stochastic and resource. Then the authors concluded that safety and reliability for multi-unit systems were complex models that involved various criteria from various dimensions.
Service placement policy, namely MinRE introduced with the aims to supply high QoS for IoT devices and to lower energy consumption in fog computing [10]. Authors classified the services into critical and normal. The goal of critical service was to reduce the responding time while normal service to lower energy consumption. MinRE organised the services in ascending order based on the deadline and priority was given based on the classified services. The policy was evaluated through simulation experiment and the results evidenced that MinRE outperformed cloud-only, edge-ward and resourceaware.

Denial of Service (DoS) attack prevention and energy conservation was an essential concern in IoT [11]. Received Signal Strength (RSS) was introduced to prevent DoS and conserved energy. RSS measured the receiving signals power to determine if the attacker on the same network using Teaching-learning-based optimization (TLBO) algorithm. Simulation results suggested that RSS was able to locate attacker within $12 \mathrm{~cm}$ and the false alarm probability was $0.7 \%$.

Load balancing mechanism depends on Jena architecture and Contract-Net Protocol (CNP) to manage the smart manufacturing equipment at the floor level posited by [12]. Firstly, resources ontology model was introduced to collect and visualise the knowledge for sharing, reusing and reasoning. Jena reasoning inputted the base model from ontology to extract the hidden information and decided the operating mode. CNP received the input from Jena reasoning to distribute the resources through three mechanisms (1) open tender, (2) bidding, and (3) winning mode.

Containerisation for resource allocation in the fog computing instead of virtual machine (VM) application is proposed by [13]. This was due to the container was more lightweight and had higher efficiency compared to VM. The concept was supported with simulation experiments where the container outperformed the VM. The authors proposed a novel task scheduling algorithm based on threshold evaluation to amplify the jobs in fog nodes and to diminish the jobs delays. However, this research did not take consideration of the fitness of cloud resources and neglected the cloud computation time.

Lightweight architecture system with cloud and edge, namely, SERENA, to implement predictive analytics platform [14]. SERENA collected the sensors data in edge gateway and processed the information in the hybrid cloud. SERENA managed the deployment in Docker service and utilised the load balancing from Docker to distribute the tasks. SERENA enabled the predictive analytic service to examine the condition of manufacturing equipment using three different machine learning algorithms: (1) decision tree, (2) gradient boosted tree, and (3) random forest.

\section{A. Critical analysis}

TABLE 1 shows the comparison of the proposed resource management technique with existing works. All of the current work only considered physical assets without considering virtual and human assets except [8]. Physical, virtual and human assets are equally crucial for the growth of business in Industry 4.0. Predictive maintenance model enables the team to fix the problem before equipment failure. However, only [14] highlights equipment predictive maintenance and resource scheduling in their work. None of the existing literature 
TABLE 1 Comparison of proposed resource management technique with existing work.

\begin{tabular}{|c|c|c|c|c|c|c|c|c|c|}
\hline \multirow[t]{2}{*}{ Work } & \multicolumn{3}{|c|}{ Asset } & \multirow{2}{*}{$\begin{array}{l}\text { Equipment } \\
\text { prediction } \\
\text { maintenance }\end{array}$} & \multirow{2}{*}{$\begin{array}{l}\text { Fog/ cloud/ } \\
\text { Local }\end{array}$} & \multicolumn{3}{|c|}{ Performance metrics } & \multirow[t]{2}{*}{ Method } \\
\hline & Physical & Virtual & Human & & & Time & Energy & Cost & \\
\hline [8] (Backman et al. 2016) & $\checkmark$ & $\checkmark$ & $\checkmark$ & & Local & & & & N/A \\
\hline [10] (Hassan et al. 2015) & $\checkmark$ & & & & Fog & $\checkmark$ & $\checkmark$ & & Policy: MinRE \\
\hline [11] Ghahramani et al. 2020) & $\checkmark$ & & $\checkmark$ & & N/A & & & & N/A \\
\hline [12] (Wan et al. 2018) & $\checkmark$ & & & & Cloud & & $\checkmark$ & & $\begin{array}{l}\text { Load balancing based on Jena } \\
\text { reasoning and CNP }\end{array}$ \\
\hline [13] (Yin et al. 2018) & $\checkmark$ & & & & Fog & $\checkmark$ & & & Containerisation \\
\hline [14] (Panicucci et al. 2020) & $\checkmark$ & & & $\checkmark$ & Fog & & & & Load balancing \\
\hline This work (proposed) & $\checkmark$ & $\checkmark$ & $\checkmark$ & $\checkmark$ & Fog & $\checkmark$ & $\checkmark$ & $\checkmark$ & GA-based \\
\hline
\end{tabular}

considered all the three performances metrics: time, energy and cost in their proposed resource management technique for physical, virtual and human assets. Owning to the reasons mentioned above, current literature becomes inefficient when solving real-life Industry 4.0 manufacturing problem where Industry 4.0 links automation, equipment, labour and software altogether and requires fog computing for low latency.

Therefore, it is necessary to build up a resource management technique that comprises of physical, virtual and human assets for Industry 4.0. Time, cost and energy of the resource management technique should be taken into consideration. The industrial big data from IIoT sensors shall be fully utilised in the predictive maintenance application. This paper addresses the challenges of existing resource management technique.

\section{PROPOSED TECHNIQUE}

This section presents a detailed description of the proposed system architecture for assets management and resource scheduling technique to handle the incoming tasks from IIoT sensors. Especially in this paper, the question is being responded: How to manage the assets in Industry 4.0 effectively using GA and astutely utilise the industrial big data to minimise manufacturing equipment failure through supervised machine learning?

\section{A. System architecture}

Fig. 1 presents the proposed system architecture of assets management. The architecture consists of five layers: asset, perception, network, fog computing and cloud computing according to their functionalities.

Asset layer contains all the resources with economic values owned by the business with the expectation to produce value. The assets are identified as primary physical, supporting physical, virtual, and human. Primary physical assets are the central elements that required for manufacturing and the manufactured products. The central elements are different manufacturing equipment and automation equipment that varies according to the nature of the industry. Supporting physical assets are the elements that enable and keep the primary manufacturing process going. Virtual assets enable digitalisation in the business and manufacturing process through the integration of IT software. Humans such as employees, vendors, customers, and end customers are the parties that directly involved in the life cycle of manufactured products. Employees are necessary to conduct manual operation, maintenance, problem-solving that cannot be replaced by automation equipment.
Perception layer consists of industrial smart sensors to gather the environmental and product information. Industrial smart sensors and meters installed in the equipment are able to detect and send physical parameters for prediction maintenance. Vision sensors are able to read the QR code and barcode which contains vulnerable information such as asset type, location, date of purchase etc. about the assets. Facial recognition eases human resource management by reducing time fraud and employees' access control.

Network layer is in charge of transmitting the real-time date from sensors to network devices, fog computing and computing layers. Business owners with multiples manufacturing plants in the whole world can be linked together to a global business through satellite communications. Wired, wireless and Intranet connections allow the communication of assets within the business.

Fog computing layer creates communication between edge devices and the cloud datacentre [15]. Fog computing is a distributed decentralised system and allows the data to send to the server to process locally. Fog computing enables real-time assets analytic applications due to the nature of low latency and bandwidth connections compared to the cloud. Cloudlet and micro cloud are small scale data centres situated at the edge of the network. The smart switch allows digital facilities management to create sustainability and greener environment. Meanwhile, the application server allows the server to run industry-specified software individually. The router at fog computing levels enables several IIoT applications such as data acquisition, smart metering and distribution automation.

Cloud computing layer allows resources management, industrial big data, and IIoT tasks processing [15]. Preprocessing, training, testing, prediction and model deployment of industrial big data are performed at this level due to the cloud offer flexibility: pay as you go. Cloud allows resources management and scheduling according to the policies of the business.

\section{B. Scheduling: Genetic Algorithm (GA) based resource scheduling technique}

Genetic Algorithm (GA) mimics Darwin's theory of evolution, where the fittest survive in nature and GA works based on state-space search. In nature, the fitter organism has a higher survival rate and able to possess their genes to the next generation through reproduction. This allows the new and fitter generation better to adapt themselves in nature. GA starts with a set of variables, where the number of chromosomes denotes as population. Their fitness value assesses new solutions (offspring). GA utilises three operators: (1) selection, (2) 

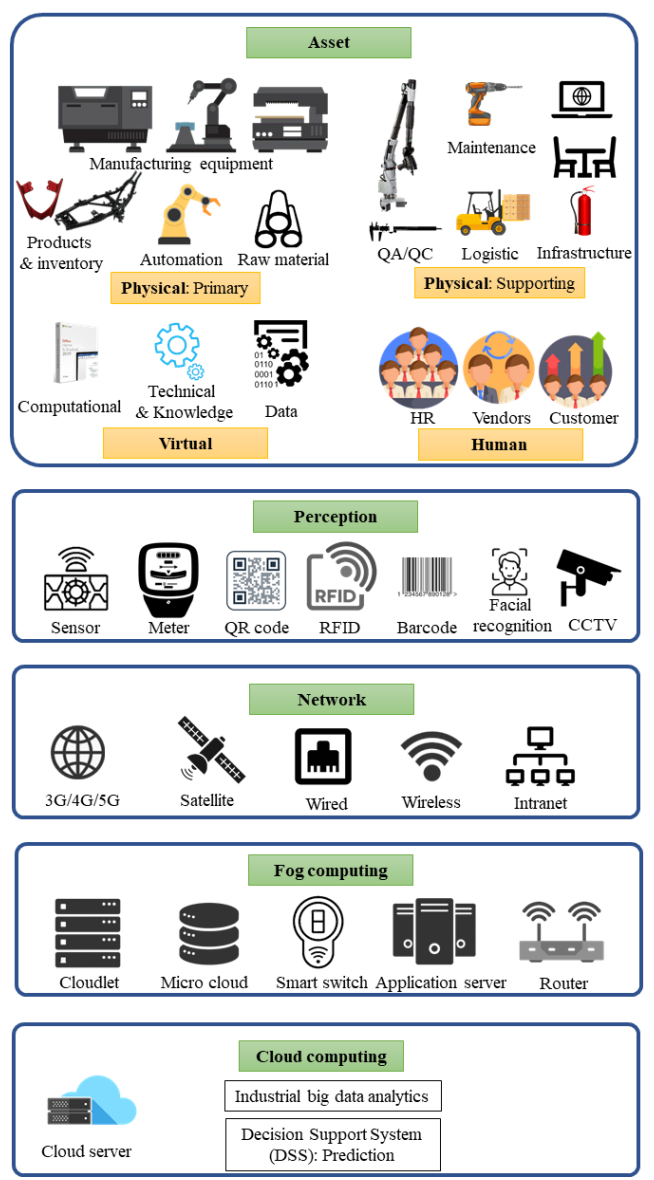

Fig. 1 System architecture.

crossover, (3) mutation to improve the solutions in each generation. The selection includes picking the parents' feature vectors from the same generation as indicated by the fitness value. Meanwhile, the main objective of crossover is to ensure the parents' genes are exchanged, and the offspring inherits the combined genes of parents. Zero cross rate implies no crossover has taken place and the offspring is an exact copy of parents. Crossover alone does not introduce a new feature vector to the offspring and possible to lead to similar solutions in the new generations. Mutations are introduced to cause random changes in the locus (positions in the chromosome). The offspring are then being placed in the new generations until the end condition is fulfilled. GA can solve resource scheduling problem due to the following characteristics:

1. GA is favourable in band selection, smaller size classification, training and testing accuracies in contrast to $\mathrm{ABC}$ and PSO.

2. GA initialises the search from population points instead of a single point.

3. GA is a direct method for global search and thus avoid trapping in local optima.

TABLE 2 presents the detailed terminology used in this research paper.

\section{PERFORMANCE EVALUATION}

To show the practicality of the proposed strategy, this section executes and deploys a case study on the present reality physical
TABLE 2 GA terminology.

\begin{tabular}{ll}
\hline $\begin{array}{l}\text { GA } \\
\text { Terminology }\end{array}$ & Description \\
\hline $\begin{array}{l}\text { Population } \\
\text { size }\end{array}$ & $\begin{array}{l}\text { The number of job requests from Ilot sensors and } \\
\text { devices. Represented by the number of chromosomes } \\
\text { in one iteration/ generation. }\end{array}$ \\
\hline $\begin{array}{l}\text { Number of } \\
\text { iterations }\end{array}$ & The number of generations \\
\hline Cross rate & $\begin{array}{l}\text { The probability of accepting a new feature vector of a } \\
\text { job }\end{array}$ \\
\hline Mutation rate & $\begin{array}{l}\text { The random probability where the elements inside the } \\
\text { feature vectors are flipped or changed. Changes caused } \\
\text { by errors while copying parents' feature vectors. }\end{array}$ \\
\hline Gene & Contained inside the feature vector \\
\hline
\end{tabular}

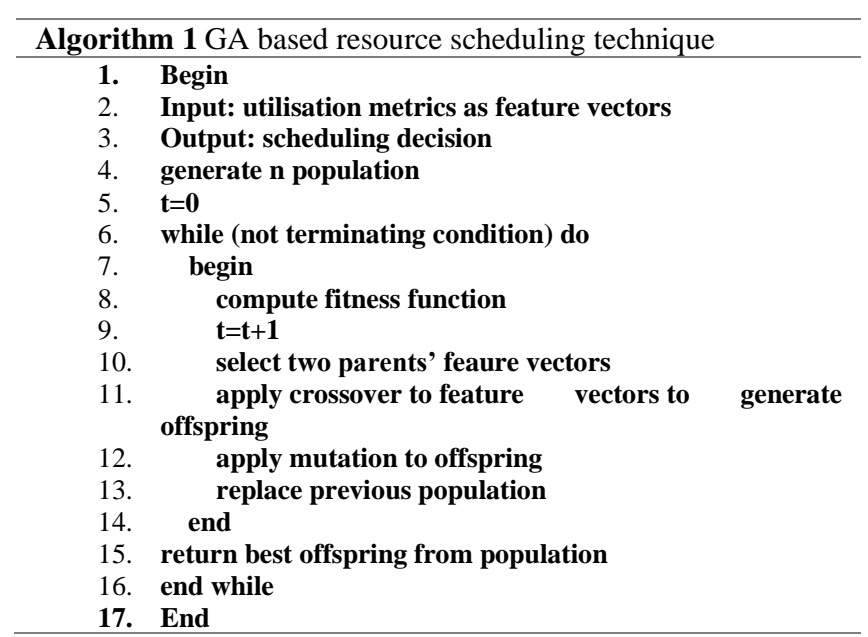

asset: manufacturing equipment predictive maintenance on FogWorkflowSim ${ }^{1}$ and Microsoft Azure Machine Learning Studio.

\section{A. Case study: Manufacturing equipment predictive maintenance}

In the manufacturing industry, manufacturing machine such as die casting machine, laser cut machine, plasma cutting machine etc. are the essential equipment to produce goods for customers. However, unforeseen machine failure and components failure can lead to production line stoppage. The domino effects of unplanned production stoppage include delay in delivery, industrial consequences links to processes, and financial losses.

The arrival of Industry 4.0 and industrial big data has created a contemporary opportunity for manufacturing equipment predictive maintenance. Predictive maintenance utilises the IIoT sensors to collect, evaluate and analyse the real-time condition of the manufacturing equipment. The benefits of predictive maintenance include maintaining high Overall Equipment Efficiency (OEE), early warning of anomalies, and awareness of the health condition of the equipment.

This case study runs on a desktop computer with configurations as described below:

- Processor: Intel Core i9-9960X CPU@ 3.10 GHz

- RAM: 64 GB

- $\quad$ System type: Windows 10 64-bit OS

\footnotetext{
${ }^{1}$ FogWorkflowSim -https://github.com/ISEC-AHU/FogWorkflowSim
} 


\section{B. Datasets}

The datasets used in this research paper are available at: Original (Highly imbalance): https://bit.ly/2VyZY5i

Amended (Under-sampling technique): https://bit.ly/37uVGS9

The datasets used for this case study is created by Fidan Boylu Uz. Due to corporate confidentiality and intellectual property, the data of attributes are represented by general word and numerical number. The dataset contains eleven attributes that identify the condition of manufacturing equipment: (1) datetime: recorded date and time, (2) machineID: physical assets identification number, (3) errorID: the error code, (4) volt: electrical voltage in Volt, (5) rotate: rotational speed, (6) pressure: measured pressure, (7) vibration: measured vibration, (8) comp: components replaced (9) model: type of equipment, (10) age: age of the equipment (11) failure: 0 or 1 . However, the dataset is highly imbalance, where it contains 6,663 samples ( $2.28 \%$ ) of class ' 1 ' and 285,006 samples $(97.72 \%$ ) of class ' 0 '. The imbalance dataset will result in high accuracy model even without training.

The dataset is then processed with the under-sampling technique to overcome the imbalance issue. Under-sampling technique randomly reduces the majority class to match the number with minority class. Consequently, the total amount of samples has reduced to 14,482 , where 6,663 samples of class ' 1 ', and 7,819 samples of class ' 0 '.

\section{Implementation of the proposed techniques in FogWorkflowSim}

In the FogWorkflowSim, the simulation environment considers four end devices (voltage sensors, pressure sensors, vibrational sensors, rotational sensors), five fog nodes (cloudlet, micro cloud, smart switch, application server, smart router) and one cloud server for this case study. The MIPS values and the execution cost of each device are as suggested in [16].

The selection of the parameters, especially cross rate and mutation rate in the GA algorithm are problem dependent. The parameters shown in TABLE 5 are tuned carefully according to previous studies [16]-[19] to optimise the performance matrices such as time, energy and cost. The performance metrics are obtained from equations (1-6) [20], [21]. The population size and number of iterations are fixed to keep the computational time low.

Execution time, $\mathbf{t}: t_{i}^{\text {total }}=t_{i}^{\text {tran }}+t_{i}^{\text {exe }}+t_{i}^{\text {rec }}+t_{i}^{\text {mig }}$

$t_{i}^{\text {tran }}$ denotes transmission time from end devices to fog server, $t_{i}^{\text {exe }}$ represents execution time at fog server, $t_{i}^{\text {rec }}$ is transmission time from fog server to end device and $t_{i}^{\text {mig }}$ refers to task migration time to different fog server because of the motion of the end device.

Cost, C: $C_{i}^{\text {total }}=T_{i}^{\text {fog }} \times C^{\text {fog }}+T_{i}^{\text {cloud }} \times C^{\text {cloud }}$

Where $T_{i}^{f o g}$ is workflow task at fog server, $T_{i}^{\text {cloud }}$ is workflow task at cloud server, $C^{\text {fog }}$ denotes unit price per second in fog server and $C^{\text {cloud }}$ represents a unit price per second in cloud computing.

Energy usage, E: $E_{i}^{\text {total }}=E_{i}^{x}+E_{i}^{y}+E_{i}^{z}$

$E_{i}^{x}=\frac{\text { Data transmission }}{\text { Bandwith }} \times P_{\text {transmission }}$

$E_{i}^{y}=\frac{\text { Task workload }}{\text { Task processing speed }} \times P_{\text {idle }}$
$E_{i}^{Z}=\frac{\text { Task workload }}{\text { Task processing speed }} \times P_{\text {end }}$

$E_{i}^{x}$ represents transmission energy from end devices to fog server, $E_{i}^{y}$ denotes idle energy utilisation of the end devices, $E_{i}^{z}$ is the load energy utilisation.

The purpose of scientific workflow is to show the dependencies between tasks and manage the data flow. Montage workflow with 60 jobs is found to be able to optimise the performance matrices.

TABLE 3 Fog environment setting in FogWorkflowSim

\begin{tabular}{llll}
\hline Parameters & $\begin{array}{l}\text { End } \\
\text { Device }\end{array}$ & Fog Nodes & $\begin{array}{l}\text { Cloud } \\
\text { Server }\end{array}$ \\
\hline Number of devices & 4 & 5 & 1 \\
\hline $\begin{array}{l}\text { Million instructions } \\
\text { per second (MIPS) }\end{array}$ & 1,000 & 1,300 & 1,600 \\
\hline Execution Cost (C\$) & 0 & 0.48 & 0.96 \\
\hline
\end{tabular}

TABLE 4 Genetic Algorithm (GA) setting in FogWorkflowSim

\begin{tabular}{ll}
\hline Parameters & Value \\
\hline Population size & 50 \\
\hline Number of iterations & 100 \\
\hline Cross rate $(\%)$ & 85 \\
\hline Mutation rate $(\%)$ & 1 \\
\hline
\end{tabular}

TABLE 5 Workflow setting in FogWorkflowSim

\begin{tabular}{ll}
\hline Parameters & Input \\
\hline Workflow type & Montage \\
\hline Total job & 60 \\
\hline
\end{tabular}

TABLE 6 Parameters for two-class logistic regression in 70:30 data splitting.

\begin{tabular}{ll}
\hline Parameters & Values \\
\hline Optimisation tolerance & 0.000100009 \\
\hline L1 Regularisation weight & 0.10009 \\
\hline L2 Regularisation weight & 0.10009 \\
\hline Memory size (MB) & 11 \\
\hline Quiet & True \\
\hline Use threads & True \\
\hline Allow unknown levels & True \\
\hline Random number seed & 12345 \\
\hline
\end{tabular}

TABLE 3 illustrates the configuration settings in the FogWorkflowSim. TABLE 4 shows the GA settings, while TABLE 5 describes the type of workflow and total job.

\section{Two-class logistic regression equipment predictive maintenance in Microsoft Azure Machine Learning}

The purpose of this case study is to predict the condition of manufacturing equipment using two class logistic regression algorithm, where 0 implies healthy equipment; meanwhile, 1 denotes equipment failure. The dataset is obtained from Section $\mathrm{IV}(\mathrm{B})$. The attributes that determined the failures are: errorID, volt, rotate, pressure, vibration, comp, age. The dataset is divided into training and testing set in the ratio of 70:30. The training dataset is used for building up the prediction algorithm while testing dataset enabled the model assessment on the realtime data. TABLE 6 describes the best parameters for two-class logistic regression after tuning. For deployment, the maintenance team can predict the equipment condition based on the real-time data input through RESTful API or Microsoft Excel. The complete workflow for this case study is available at https://bit.ly/2I5uP6u. 


\section{EXPERIMENTAL RESULTS}

\section{A. Performance of GA in FogWorkflowSim}

Fig. 2 compares the (a) execution time, (b) operating cost, (c) energy usage of GA with MinMin, MaxMin, FCFS, RoundRobin scheduling algorithm.

(a) Execution time: The execution time of GA is $28.1 \%$, $9.1 \%, 0.5 \%, 9 \%$ faster compared to MinMin, MaxMin, FCFS, RoundRobin respectively. The execution time of GA is much lower is due to the flexibility of parameters tuning. Crossover is the most important operation and enables the good characteristics of the individual parents to recombine. The algorithm can discover the solution more efficiently throughout the acceleration in each generation and has the lowest execution time among all. Furthermore, the execution time of GA is lower than heuristic algorithm e.g. RoundRobin despite the complexity of GA is due to job scheduler in CloudSim. When the user sends a high number of job requests to the cloudlet simultaneously, CloudSim is not able to execute them and job scheduler places the requests in a queue system. Job scheduler positions and executes the job request from the lowest execution time to the highest and send back to the user. This leads to lower execution times of the GA method.

(b) Cost: The cost of GA is $28.1 \%, 94.2 \%, 93.8 \%, 93.7 \%$ lower compared to MinMin, MaxMin, FCFS, RoundRobin respectively. GA optimises the distribution of tasks and has a better fitness value. Thus this further reduces the executing cost.

(c) Energy usage: The energy usage of GA is $3.9 \%$, $11.6 \%, 5.6 \%, 20.7 \%$ lesser compared to MinMin, MaxMin, FCFS, RoundRobin respectively. Montage workflow allows label-based clustering, where the same tasks in the workflow can cluster together. Instead of executing individual workload, clustered workload minimises the network traffic and further reduces energy usage. Furthermore, GA strategy tends to club computationally expensive tasks in resourceintensive cloud nodes and simpler tasks in edge devices. This leads to a reduction in energy usage.

\section{B. Training and testing accuracy of predictive maintenance}

The equipment predictive maintenance employs two-class logistic regression to predict the health of manufacturing equipment. Two-class logistic regression allows good accuracy with fast training time [22]. Fig. 3 depicts the Receiver Operating Characteristic (ROC) curve of training and testing dataset. Setting the threshold as a constant value of 0.5 in both training and testing dataset, the Area Under Curve (AUC) of ROC is 0.990 and 0.987 , respectively. AUC closer to 1 represents a better measure of separability, whist $\mathrm{AUC}=1$ is where classifier impeccably recognises all positive and negative classes accurately. The training accuracy and testing accuracy of the model is $95.1 \%$ and $94.5 \%$, respectively. TABLE 7

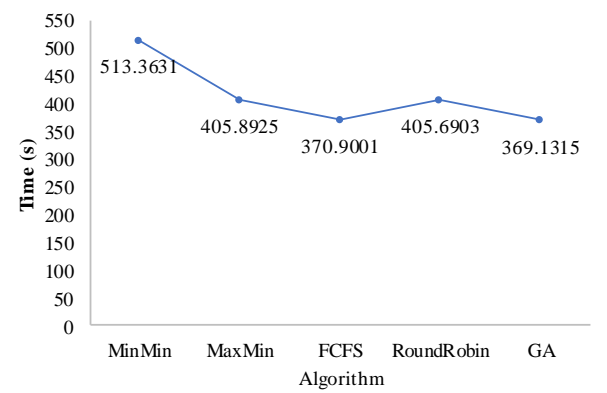

(a)

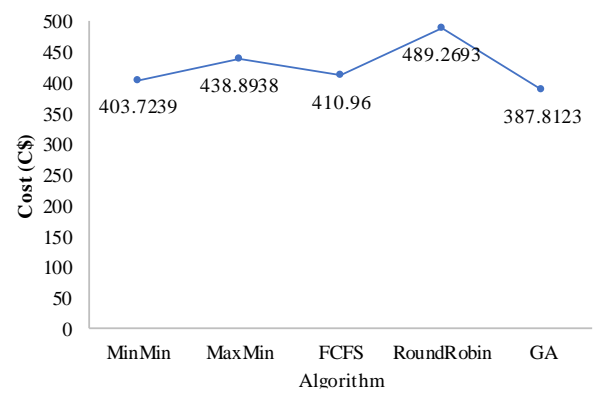

(b)

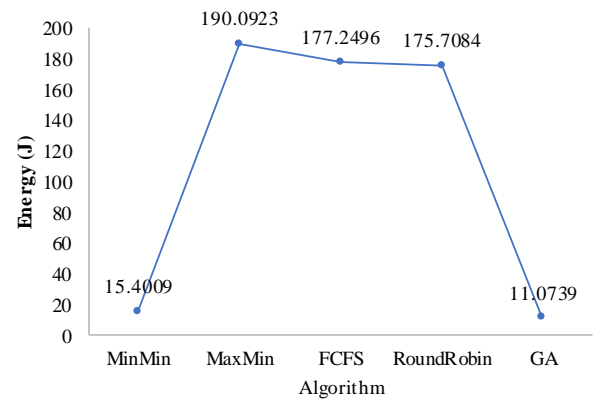

(c)

Fig. 2 Evaluation results for MinMin, MaxMin, FCFS, RoundRobin, GA: (a) Time, (b) Cost, (c) Energy

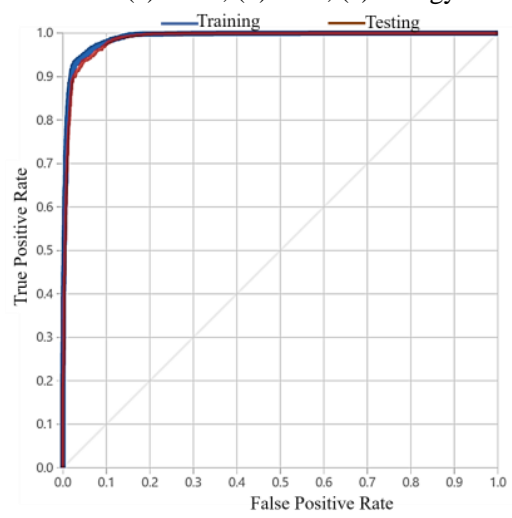

Fig. 3 Training and testing of two-class logistic regression ROC curve.

TABLE 7 Confusion matrix and measure of training and testing.

\begin{tabular}{|c|c|c|c|c|c|}
\hline \multirow{2}{*}{ 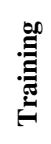 } & $\begin{array}{c}\mathrm{TP} \\
4412\end{array}$ & $\begin{array}{l}\text { FN } \\
274\end{array}$ & $\begin{array}{c}\text { Accuracy } \\
0.951\end{array}$ & $\begin{array}{c}\text { Precision } \\
0.952\end{array}$ & $\begin{array}{c}\text { Threshold } \\
0.5\end{array}$ \\
\hline & $\begin{array}{l}\text { FP } \\
223\end{array}$ & $\begin{array}{c}\mathrm{TN} \\
5228\end{array}$ & $\begin{array}{c}\text { Recall } \\
0.942\end{array}$ & $\begin{array}{c}\text { F1 Score } \\
0.947\end{array}$ & $\begin{array}{l}\text { AUC } \\
0.990\end{array}$ \\
\hline \multirow{2}{*}{ 苞 } & $\begin{array}{c}\mathrm{TP} \\
1844\end{array}$ & $\begin{array}{l}\text { FN } \\
133\end{array}$ & $\begin{array}{c}\text { Accuracy } \\
0.945\end{array}$ & $\begin{array}{c}\text { Precision } \\
0.946\end{array}$ & $\begin{array}{c}\text { Threshold } \\
0.5\end{array}$ \\
\hline & $\begin{array}{l}\text { FP } \\
105\end{array}$ & $\begin{array}{c}\mathrm{TN} \\
2263\end{array}$ & $\begin{array}{c}\text { Recall } \\
0.933\end{array}$ & $\begin{array}{c}\text { F1 Score } \\
0.939\end{array}$ & $\begin{array}{l}\text { AUC } \\
0.987\end{array}$ \\
\hline
\end{tabular}


shows the confusion matrix along with their measures of training and testing dataset.

\section{CONCLUSIONS AND FUTURE WORK}

This paper proposed the Genetic Algorithm (GA) as the technique for resource management in assets management application for Industry 4.0. Proposed system architecture contains five layers, including (1) assets, (2) perception, (3) network, (4) fog computing and (5) cloud computing. GA was evaluated along with MinMin, MaxMin, FCFS and RoundRobin in FogWorkflowsim to show the effectiveness of the proposed technique. The performance metrics for the evaluation were execution time, cost and energy. Extensive simulation experiment evidenced that GA outperformed MinMin, MaxMin, FCFS and RoudRobin in terms of having the lowest execution time, cost and energy. The execution time was $0.48 \%$ faster, the cost was $5.43 \%$ lower and energy usage was $28.10 \%$ lower in comparison to second-best results. Lastly, a model for equipment predictive maintenance had been deployed using a supervised machine learning algorithm, twoclass logistic regression. The model was able to predict if the manufacturing equipment failing and produced an early warning alert for the production line. The training accuracy and testing accuracy for the model were $95.1 \%$ and $94.5 \%$ each.

\section{A. Future work}

In spite of the fact that the proposed resource management technique demonstrated efficiency and able to distribute the tasks effectively, it very well may be additionally enhanced in a broader scope followed by the accompanying viewpoints:

1. Reliability and security communication: There is a need to ensure up-to-date industrial security communication among the devices to prevent cyberattacks.

2. Performance metrics: The performance metrics of the simulation can further include network latency, network bandwidth, jitter.

3. Extending to varies domains: Current paper focuses on the manufacturing industry. This can be extended to varies domains of Industry 4.0 such as construction, oil and gas, chemical due to the beneficial of asset usage, quality control, supply chain management, product monitoring, work environment wellbeing.

\section{REFERENCES}

S. S. Gill et al., "Transformative effects of IoT, blockchain and artificial intelligence on cloud computing: Evolution, vision, trends and open challenges," Internet of Things, vol. 8, 2019.

[2] M. S. de Brito, S. Hoque, R. Steinke, A. Willner, and T. Magedanz, "Application of the Fog computing paradigm to Smart Factories and cyber-physical systems," Trans. Emerg. Telecommun. Technol., vol. 29, no. 4, pp. 1-14, 2018.

[3] D. Mourtzis, E. Vlachou, and N. Milas, "Industrial Big Data as a Result of IoT Adoption in Manufacturing," Procedia CIRP, vol. 55, pp. 290-295, 2016.

[4] S. Tuli et al., "HealthFog: An ensemble deep learning based Smart Healthcare System for Automatic Diagnosis of Heart Diseases in integrated IoT and fog computing environments," Futur. Gener. Comput. Syst., vol. 104, pp. 187-200, 2020.

[5] S. Poslad, Ubiquitous Computing. Chichester, UK: John Wiley \& Sons, Ltd, 2009.

[6] S. Singh and I. Chana, "A Survey on Resource Scheduling in Cloud Computing: Issues and Challenges," J. Grid Comput., vol. 14, no. 2, pp. 217-264, Jun. 2016.

[7] J. Alcaraz and C. Maroto, "A Robust Genetic Algorithm for Resource Allocation in Project Scheduling," Ann. Oper. Res., vol. 102, no. 14, pp. 83-109, 2001.

[8] J. Backman, J. Vare, K. Framling, M. Madhikermi, and O. Nykanen, "IoT-based interoperability framework for asset and fleet management," IEEE Int. Conf. Emerg. Technol. Fact. Autom. ETFA, vol. 2016-Novem, pp. 0-3, 2016.

[9] S. Petchrompo and A. K. Parlikad, "A review of asset management literature on multi-asset systems," Reliab. Eng. Syst. Saf., vol. 181, no. September 2018, pp. 181-201, 2019.

[10] H. O. Hassan, S. Azizi, and M. Shojafar, "Priority, network and energy-aware placement of IoT-based application services in fogcloud environments," IET Commun., vol. 14, no. 13, pp. 2117-2129, 2020

[11] M. Ghahramani, R. Javidan, M. Shojafar, R. Taheri, M. Alazab, and R. Tafazolli, "RSS: An energy-efficient approach for securing IoT service protocols against the DoS attack," IEEE Internet Things J., vol. XX, no. XX, pp. 1-1,2020.

[12] J. Wan et al., "Toward dynamic resources management for IoT-based manufacturing," IEEE Commun. Mag., vol. 56, no. 2, pp. 52-59, 2018

[13] L. Yin, J. Luo, and H. Luo, "Tasks Scheduling and Resource Allocation in Fog Computing Based on Containers for Smart Manufacturing," IEEE Trans. Ind. Informatics, vol. 14, no. 10, pp. 4712-4721, 2018.

[14] S. Panicucci et al., "A cloud-to-edge approach to support predictive analytics in robotics industry," Electron., vol. 9, no. 3, pp. 1-22, 2020.

[15] S. S. Gill, P. Garraghan, and R. Buyya, "ROUTER: Fog enabled cloud based intelligent resource management approach for smart home IoT devices," J. Syst. Softw., vol. 154, pp. 125-138, Aug. 2019.

[16] X. Liu et al., "FogWorkflowSim: An Automated Simulation Toolkit for Workflow Performance Evaluation in Fog Computing," in 2019 34th IEEE/ACM International Conference on Automated Software Engineering (ASE), 2019, pp. 1114-1117.

[17] M. Obitko, "Introduction to Genetic Algorithms," 1998. [Online]. Available:

https://courses.cs.washington.edu/courses/cse473/06sp/GeneticAlg Demo/main.html. [Accessed: 01-Aug-2020].

[18] M. Birattari, Tuning Metaheuristics, 197th ed., vol. 197, no. 1. Berlin, Heidelberg: Springer Berlin Heidelberg, 2009.

[19] H. M. Pandey, A. Chaudhary, and D. Mehrotra, "A comparative review of approaches to prevent premature convergence in GA," Appl. Soft Comput. J., vol. 24, pp. 1047-1077, 2014.

[20] L. Fan, X. Liu, X. Li, D. Yuan, and J. Xu, "Graph4Edge: A Graphbased Computation Offloading Strategy for Mobile-Edge Workflow Applications," 2020 IEEE Int. Conf. Pervasive Comput. Commun. Work. PerCom Work. 2020, no. 61972001, pp. 3-6, 2020.

[21] J. Xu et al., "Mobility-Aware Workflow Offloading and Scheduling Strategy for Mobile Edge Computing," in Lecture Notes in Computer Science (including subseries Lecture Notes in Artificial Intelligence and Lecture Notes in Bioinformatics), vol. 11945 LNCS, 2020, pp. 184-199.

[22] C.J. Gronlund, Josée Martens, and Francesca Lazzeri, "How to select a machine learning algorithm," Microsoft , 05-Jul-2020. [Online]. Available: https://docs.microsoft.com/en-us/azure/machinelearning/how-to-select-algorithms. [Accessed: 10-Aug-2020].

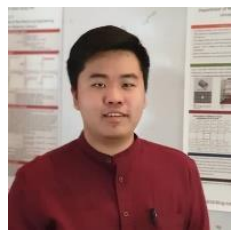

Yyi Kai Teoh is an MSc student at Queen Mary University of London, UK. Currently, he is completing his industrial placement at an IoT consulting company in London, working as IoT \& Hardware Systems Analyst. Yyi Kai has BEng (Hons) in Mechanical Engineering from the University of Nottingham, UK. His research interests include IoT, Machine Learning, Distributed Systems and Cloud Computing. 


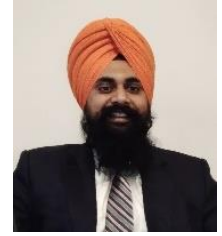

Sukhpal Singh Gill is a Lecturer (Assistant Professor) in Cloud Computing at School of EECS, Queen Mary University of London, UK. Prior to this, Dr. Gill has held positions as a Research Associate at the School of Computing and Communications, Lancaster University, UK and also as a Postdoctoral Research Fellow at CLOUDS Laboratory, The University of Melbourne, Australia. His research interests include Cloud Computing, Fog Computing, Internet of Things and Energy Efficiency.

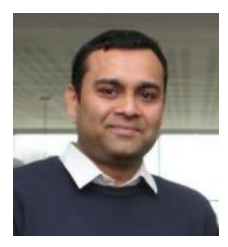

Ajith Kumar Parlikad is Reader in Asset Management at Cambridge University Engineering Department. He is based at the Institute for Manufacturing, where he is the Head of the Asset Management research group. He is a Fellow and Tutor at Hughes Hall. Ajith joined Cambridge University to read for his $\mathrm{PhD}$ degree, which he successfully completed in August 2006. Ajith's current research focusses on the development and exploitation of digital twins of complex asset systems bringing together data from disparate sources to improve asset management. 